\title{
A well differentiated oral squamous cell carcinoma involving the lateral border of tongue- A unique case report
}

\author{
Vammi Sirisha
}

MDS (Oral Medicine and Radiology), Private Practitioner, Bangalore, Karnataka, India

*Corresponding Author: Vammi Sirisha

Email: luckycherry.siri@gmail.com

\begin{abstract}
Oral cancer is the $6^{\text {th }}$ commonest among all the malignancies, affecting more than half a million people each year, with a reported of 75000 80000 new cases annually in India. Among other Head and Neck malignancies, 95\% of cases accounts for oral squamous cell carcinomas. According to the surveillance, Epidemiology and End results program stated that the relative prognosis rate of $62.2 \%$ is given with an overall period of 5 years. Oral squamous cell carcinoma is before to specific potentially malignant disorders, of which the most common ones are Oral leukoplakia and OSMF due to a well-known risk factors like consumption of tobacco, arecanut and alcohol. Other factors include age and family history of cancer, HPV infections, immunosuppression, UV light and some hereditary associated conditions etc. According to WHO prognosticate by 2020, the tobacco-related deaths may exceeds 1.5 million per year. Several epidemiological studies showed that smokers tends to prosper oral cancer 5-9 times than non-smokers. This case report describes a well-differentiated oral squamous cell carcinoma involving the left lateral border of tongue.
\end{abstract}

Keywords: OSCC (Oral Squamous Cell Carcinoma), OSMF (Oral Submucous Fibrosis), EGFR (Epithelial Growth Factor Receptor), LOH (Loss of Heterozygosity), CGH (Comparative Genomic Hybridization).

\section{Introduction}

Cancer is the second leading cause of death globally after cardiovascular diseases and oral cancer is the third most common types of cancers affecting the mankind worldwide after lung and breast cancer. ${ }^{1}$ The overall prevalence of oral cancer accounts for approximately $3 \%$ of all malignancies and estimated 2,70,000 people are annually attended. ${ }^{2}$ The prevalence in south-east Asian countries like India is much higher than the worldwide prevalence. $^{3}$ The latest statistics see an increased incidence of oral cancers in India by a whopping $114 \%$ in the past six years ${ }^{4}$. The number of incident lip and oral cavity cancer cases in India in 2016 was 1,13,000 and the prevalent cases were 3,97,000. In India, the Lip and oral cavity cancer was the most common incident cancers in males with a maximum prevalence in the states of Madhya Pradesh, followed by Gujarat and kerala. ${ }^{5}$

Most of the oral cancers (92-95\%) comprises of oral squamous cell carcinomas. According to the International classification of diseases of the Head and neck malignancies, oral cancer is considered as a subgroup which may involves tongue, lips, salivary glands, gingiva, floor of the mouth, oropharynx, buccal surfaces and other intraoral locations, ${ }^{6}$ commonly affecting the low income communities and in older individuals. Moreover, $90 \%$ of being in the over 45 years age group who are exposed to the known risk factors of tobacco and alcohol. Clinically,
OSCC includes lip cancer, which accounts for the majority of OSCC and intraoral cancer which mainly affects the tongue. ${ }^{7}$ OSCC within the mouth affects mainly the tongue, particularly the lateral borders of tongue, in older male individuals and are often related to lifestyle habits which are largely tobacco and alcohol related. ${ }^{8}$

Several literatures stated that smoking and alcohol were considered as a major risk factors in $90 \%$ of cases, having them both a synergic affect. ${ }^{9}$ The development of OSCC is considered as a complex multiple process, in which normal oral mucosal keratinocytes are chronically exposed to these risk factors may lead to the imbalance in homeostasis and generate genetic instability. ${ }^{10}$

This case report presents a patient with a chronic nonhealing ulcer which turned out to be a well differentiated oral squamous cell carcinoma is discussed. He had the habit of both smoke and smokeless tobacco usage and alcohol.

\section{Case Report}

A 55 year old male patient visited with the chief complaint of an ulcer on the left side of tongue since 5 months. History of present illness revealed that the ulcer was associated with pain and mild burning sensation since 5 months, that gradually increased in intensity, aggrevating on taking spicy foods. No history of fever, trauma, loss of appetite. History revealed that he was a chronic smoker 
and had habit of alcohol consumption for the past 30 years and habit of pan chewing (gutkha) for the past 10 years. Patient referred to the tobacco cessation centre for counselling. During routine investigations, there was no relevant medical history. On extraoral examination, no facial asymmetry was observed. Solitary right and left submandibular lymph nodes were palpable of size $1 \times 1 \mathrm{~cm}$, roughly oval in shape, tender, freely movable and firm in consistency. Level 2 lymph nodes palpable on left side, mildly tender and freely movable.

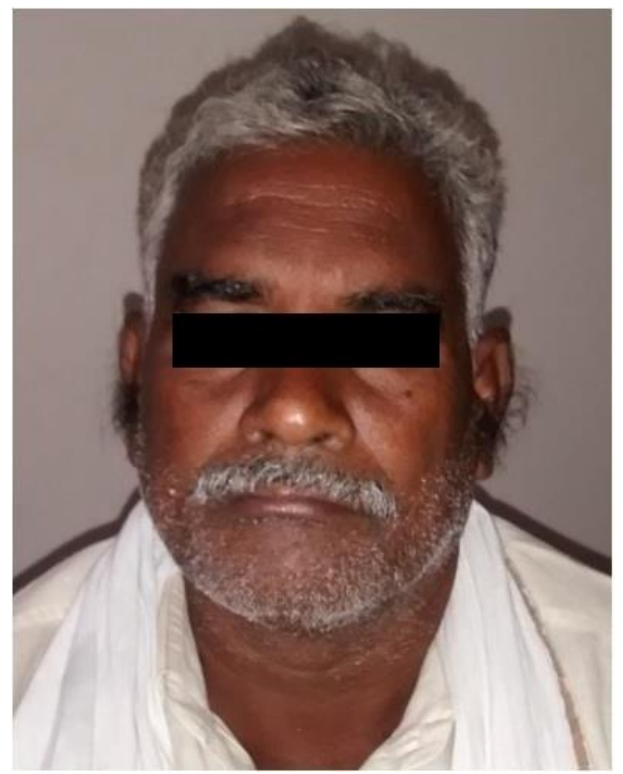

Fig 1: Frontal view of the patient

On intraoral examination, blanching of labial mucosa and buccal mucosa with diffuse melanin pigmentation is seen. Restricted mouth opening of $25 \mathrm{~mm}$ present. A solitary ulcero-infiltrative, erythematous lesion of size approximately $2.5 \mathrm{~cm}$, roughly oval in shape seen on the left lateral border of the tongue extending anterioposteriorly from $2 \mathrm{~cm}$ from the tip of the tongue and $3 \mathrm{~cm}$ away from the posterior $1 / 3^{\text {rd }}$ of tongue. Superioinferiorly, extending from the dorsum of the tongue till $0.5 \mathrm{~cm}$ away from the floor of the mouth. Margins are raised. Floor of the ulcer anteriorly erythematous and posteriorly covered with yellowish pseudomembranous slough. No discharge is seen. Tongue shows restricted movements. On palpation, the lesion is tender and firm is consistency. Edges are raised and everted, base is indurated. Vertical fibrous bands are palpable on the right and left buccal mucosa along with the labial bands with leathery texture.

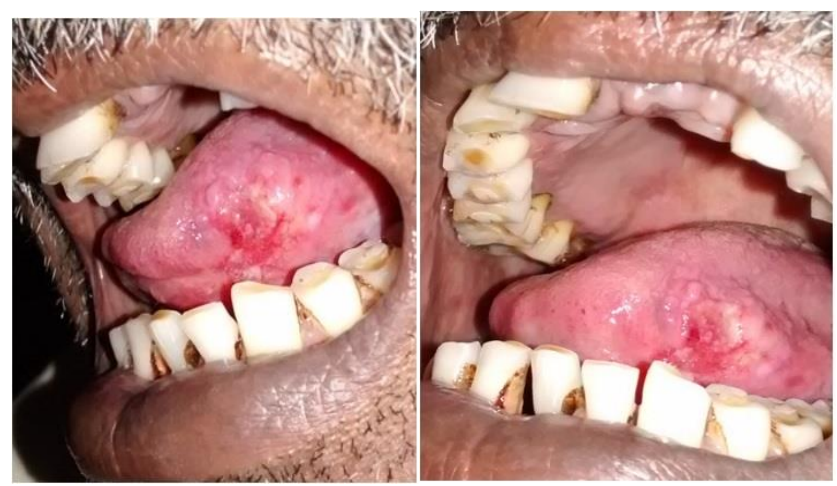

Fig. 2 \& 3: Showing an ulcero-infiltrative lesion on the left lateral border of the tongue.

Provisional diagnosis of malignant ulcer on the left lateral border of tongue. Clinical staging of tumor T1N2M0 with OSMF grade 4A is given. Chair side investigations was carried out by applying toluidine blue stain which stained and lateral incisional biopsy was performed under local anaesthesia and specimen sent for histopathologic evaluation. The H\&E stained sections shows formation of keratin pearls within the connective tissue. Neoplastic epithelial cells are seen in the connective tissue and the result was a well differentiated squamous cell carcinoma on the left lateral border of tongue.

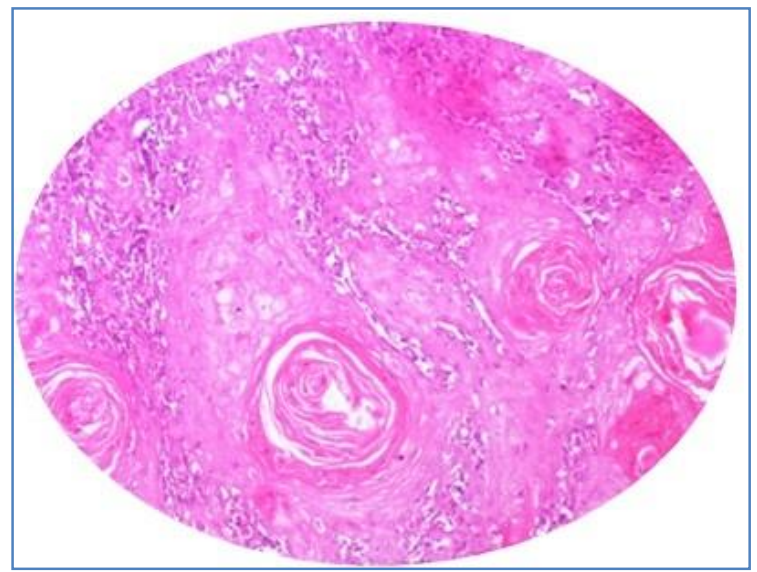

Fig. 4: Low power resolution shows islands of malignant squamous cells

On other investigations, ultrasound of neck revealed evidence of small $5-8 \mathrm{~mm}$ non specific lymphnodes in bilateral level 2 regions. Patient was referred to cancer hospital for further evaluation and management. Hemiglossectomy with radical neck dissection was done. 


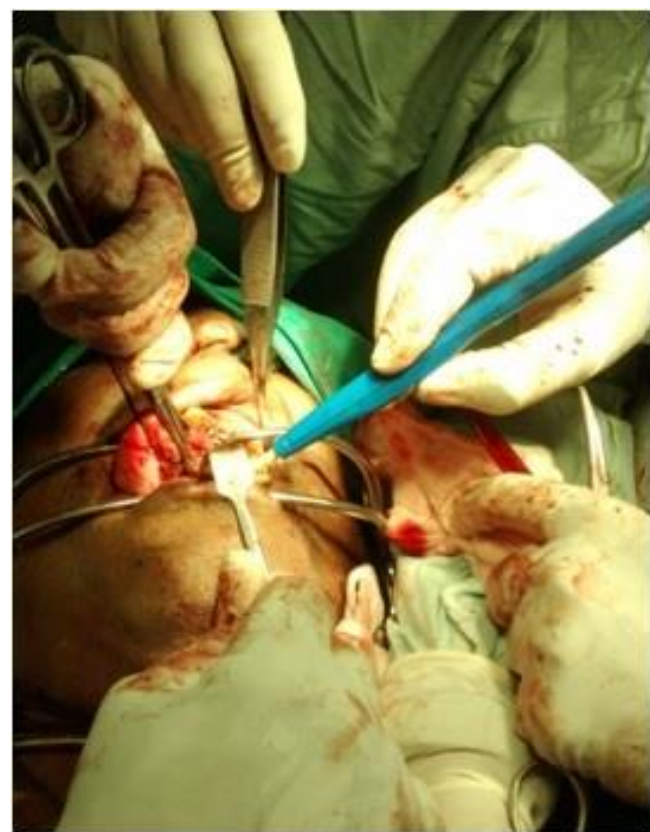

Fig. 5: Operative procedures

Patient is currently under periodic control, including a follow-up by a speech therapist and nutrionist.

\section{Discussion}

The overall incidence and mortality attributed to the OSCC is increasing with current estimates of age standardized incidence and mortality of 6.6/100,000 and $3.1 / 100,000$ in men and 2.9/100,000 and 1.4/100,000 in women. ${ }^{11}$ The survival index continues to be $50 \%$ as compared to the progress in diagnosis and treatment of other malignant tumors. The early detection of precancerous and cancerous lesions to be improved for effective treatment and minimal morbidity. ${ }^{12}$ The risk factors include tobacco associated intraoral carcinogens, which may play a synergistic role in oral tumorigenesis. ${ }^{13}$ The other risk factors includes the alcohol consumption, low socio-economic conditions related to the poor oral hygienic, poor diet and rampant viral infections. ${ }^{14}$

On the other hand, the molecular pathogenesis of OSCC is a multiple process. The genetic alterations observed in the head and neck cancers are mainly due to oncogenic activation and tumor suppression genes inactivation leading to de regulation of cell proliferation and death. ${ }^{15}$ These genetic alterations include gene amplification and over expression of oncogenes such as myc, erb-2, epidermal growth factor receptor (EGFR), cyclinD1 and mutations, deletions and hypermethylation leading to p16 and p53 tumor suppressor gene inactivation. ${ }^{16}$ The evaluation of the genomic stability can be done using technique such as a loss of heterozygosity (LOH) screening and comparative genomic hybridization $(\mathrm{CGH}) .{ }^{17}$ In majority of cancers, highly vascularised tumors showed a poor prognosis and the influence of tumor angiogenesis proved to be independent of conventional prognostic indicators. ${ }^{18}$

OSCC affecting tongue is most commonly of epithelial origin and may result from the chronic irritation. According to site, the tongue lesions constitues $34.6 \%$ with the lateral borders, being the most frequently affected site. ${ }^{19}$ Well differentiated tumors has better prognostic value than poorly differentiated with a lower probability of lymph node metastasis. OSCC of the oral tongue is rare in young adults. Friedlander et al in a study of younger and older patients with tongue cancer matched for alcohol and tobacco usage..$^{20}$ Kurikose et al in their study comparing the tongue cancer in young and older OSCC patients in India concluded that in younger patients, OSCC of tongue was associated with few etiological factors and in older patents, it was always seen in association with smoking, alcohol and chewing. Metastastic spread of OSCC of tongue is facilitated by tongues rich lymphatic network, which tends to increase with the size of the primary tumor.

Lymphnode metastasis is a well known negative prognostic factor for OSCC of tongue and decreases the survival rate by $50 \%$. Several studies have shown that the survival rate in men are lower than women with tongue cancer. The reported incidence of local recurrence rates in the margins ranged from $29 \%-70 \%$ and were $4 \%-38 \%$ for negative resection margins. ${ }^{21}$

\section{Conclusion}

The poor prognosis of head and neck squamous cell carcinomas has intensified current research efforts in the field of prevention and early detection. The knowledge of analysing new genetic alterations will helpful for newer diagnostic and prognostic factors, which are helpful for rational and effective management of the patients.

\section{Source of funding}

None.

\section{Conflict of interest}

None.

\section{References}

1. GBD 2016 Cancer collaborators. Global, regional and national cancer incidence, mortality, years of life lost, years lived with 
disability and disability- adjusted life years for 29 cancer groups, 1990 to 2016: a systemic analysis for the Global Burden Of Disease Study. JAMA Oncol 2018; published online June 2. DOI:10.1001/jamaoncol.2018.2706.

2. Mishra A, Verma M. Cancer biomarkers: Are we ready for the prime time cancers 2010;2:190-208.

3. Mortazavi H, Baharvand M, Mehdipour M. Oral potentially malignant disorders: an overview of more than 20 entities. $J$ Dent Res Dent Clin Dent Prospects 2014;8(1):6-14.

4. Lohe V, Degwekar S, Bhowate R, Kadu R, Dangore S, Evaluation of correlation of serum lipid profile in patients with oral cancer and precancer and its association with tobacco abuse. J Oral Pathol Med 2010;39:141-8.

5. India State-Level Disease Burden Initiative Collaborators. Nations within a nation: variations in epidemiological transition across the states of India, 1990-2016 in the Global Burden of Disease study. Lancet 2017;390;243760.

6. Speight PM. Update on Oral epirhelial dysplasia and progression to cancer. Head Neck Pathol 2007;1:61-6.

7. Scully C, Bagan J. Oral squamous cell carcinoma: overview of current understanding of aetiopathogenesis and clinical implications. Oral Dis 2009;15:388-99.

8. Silverman S.Demographics and occurrence of oral and pharyngeal cancers. The outcomes, the trends, the challenges. $J$ Am Dent Assoc 2001;132 suppl: 7S-11S.

9. Dissanayaka WL, Pitiyage G, Kumarasiri PV, Liyanage RL, Dias KD and Tilakarantne WM. Clinical and Histopathologic parameters in survival of oral squamous cell carcinoma. Oral Surg Oral Med Oral Pathol Oral Radiol 2012;113:518-25.

10. Koontongkaew $\mathrm{S}$. The tumor microenvironment contribution to development, growth, invasion and metastasis of head and neck squamous cell carcinomas. J Cancer 2013;4:66-8.

11. Landis SH, Murray T, Bolden S, Wingo PA, Cancer stastistics, 1999. CA Cancer J Clin 1999;49:8-31.
12. Mehrotra R, Gupta A, Singh M, Ibrahim R. Application of cytology and molecular biology in diagnosis premalignant or malignant lesions. Mol Cancer 2006;5:11.

13. Walker DM, Boey G, Mc Donald LA. The pathology of oral cancer. Pathol 2003; 35:376-83.

14. Franceschi S, Bidoli P, Herrero R, Munoz N. Comparision of cancers of the oral cavity and pharynx world wide: eiological clues. Oral Oncol 2000;36:106-15.

15. Mehrotra R, Vasstrand EN, Ibrahim SO. Recent advances in understanding carcinogenicity of oral squamous cell carcinoma. From basic molecular biology to latest genomic and proteomic findings. Cancer Gen Proteom 2004;1:283-94.

16. Weinberg RA. Tumor suppreessor genes. Sci 1991;254:113846;52.

17. Bergers G, Benjamin LE. Tumorigenesis and the angiogenic switch. Nat Rev Cancer 2003;3:401-10.

18. Kerbel RS, Tumor angiogenesis: Past, present and the near future carcinogenesis 2000;21:505-15.

19. Mehta R. A case report: Oral squamous cell carcinoma of Anterolateral Border of the Tongue. J Dent Sci 2013;3(1):32-4.

20. Friedlander PL, Schantz SP, Shaha AR, Yu G, Shah JP. Squamous cell carcinoma of thr tongue in young patients: A matched-pair analysis. Head Neck 1998;20:363-8.

21. Mildasuslu, Ali Sefik Hosal, Tugba Aslan, Buleent Sozeri, Anil Dolgunpd. Carcinoma of the oral tongue: A case series analysis of prognostic factors and surgical outcomes. J Oral Maxillfacsurg 2013;71:1283-90.

How to cite this article: Sirisha V. A well differentiated oral squamous cell carcinoma involving the lateral border of tongue- A unique case report. Int Dent J Student Res 2019;7(4):121-4. 